\title{
Generation and Detection of 28 Gbaud Polarization Switched-QPSK in WDM Long-Haul Transmission Systems
}

\author{
Jérémie Renaudier, Member, IEEE, Paolo Serena, Alberto Bononi, Member, IEEE, \\ Massimiliano Salsi, Member, IEEE, Oriol Bertran-Pardo, Member, IEEE, Haïk Mardoyan, \\ Patrice Tran, Eric Dutisseuil, Gabriel Charlet, Member, IEEE, and Sébastien Bigo, Member, IEEE
}

\begin{abstract}
We report on an experimental long-haul transmission of a 28 Gbaud PS-QPSK signal in WDM scenarios. This report presents, to the authors' knowledge, a novel implementation of an optical coherent receiver for the polarization-switched quadrature phase shift keying (PS-QPSK) modulation format, with minor variations on the standard coherent PDM-QPSK receiver. We compare the performance of PS-QPSK with that of PDM-QPSK, over a WDM system based on $100 \mathrm{~Gb} / \mathrm{s}$ PDM-QPSK and standard single mode fiber (SSMF), and we demonstrate its interest for software-defined optical transceivers operating over dispersion managed and non-managed transmission links.
\end{abstract}

Index Terms-Coherent communications, digital communications, four-dimensional (4-D) constellations, optical fiber communications, polarization-switched quadrature phase-shift keying (QPSK).

\section{INTRODUCTION}

$\mathbf{F}$ OUR-DIMENSIONAL signaling featuring the combined use of real and imaginary components of light and its two orthogonal polarization states has been recently proposed [1]-[3]. Among all the modulation formats proposed for optical transmission, the polarization switched (PS-) quadrature phase shift keying (QPSK) format has attracted much attention because it is viewed to be the most power-efficient [2]. Indeed some theoretical considerations predict that PS-QPSK should exhibit a greater sensitivity to optical noise than that of polarization division multiplexed (PDM)-QPSK. This improvement in sensitivity to optical noise has been recently confirmed in a single channel experiment realized at 10 Gbaud [4]. Moreover, numerical investigations have also reported an improved tolerance to nonlinearities in wavelength division multiplexed (WDM) transmission scenarios at a bit rate of $100 \mathrm{Gbit} / \mathrm{s}$ [5],

Manuscript received September 16, 2011; revised December 09, 2011; accepted January 09, 2012. Date of publication January 23, 2012; date of current version April 04, 2012.

J. Renaudier, M. Salsi, O. Bertran-Pardo, H. Mardoyan, P. Tran, E. Dutisseuil, G. Charlet, and S. Bigo are with Alcatel-Lucent Bell Labs, 91620 Nozay, France (e-mail: jeremie.renaudier@alcatel-lucent.com).

P. Serena and A. Bononi are with the Dipartimento di Ingegneria dell'Informazione, Università degli Studi di Parma, 43100 Parma, Italy.

Color versions of one or more of the figures in this paper are available online at http://ieeexplore.ieee.org.

Digital Object Identifier 10.1109/JLT.2012.2185682
[6]. Despite these advantages, PS-QPSK is not as spectrally efficient as PDM-QPSK, which makes it less adequate for optically-routed $100 \mathrm{~Gb} / \mathrm{s}$ networks. Nevertheless, PS-QPSK may be a very attractive complement in the list of conventional modulation formats to be used in adaptive bit-rate networks. In such networks, the data rate of each light path is dynamically adapted to both the capacity demand and the amount of physical impairments to be overcome [7]. Operating with the same hardware and baud rate as PDM-QPSK, PS-QPSK offers an interesting fall-back alternative to PDM-QPSK. Typically, over links where propagation impairments are strong, PDM-QPSK data may not reach their destination without intermediate $\mathrm{O} / \mathrm{E} / \mathrm{O}$ regeneration, whereas PS-QPSK format could survive the same impairments at the expense of $25 \%$ data-rate reduction [6].

We report here on a series of experiments conducted at 28 Gbaud using single carrier coherent PS-QPSK, expanding upon our initial results [8]. We propose a novel method for generating and detecting PS-QPSK signals compatible with the widely used constant modulus algorithm (CMA) in coherent PDM-QPSK systems. We compare the performance of PS-QPSK to that of PDM-QPSK operating at the same baud rate over a WDM system based on $100 \mathrm{~Gb} / \mathrm{s}$ PDM-QPSK and standard single mode fiber (SSMF), using the same transmitter hardware configuration and only minor adjustments of the DSP architecture at the receiver side. We also analyze the impact of electrical and optical dispersion compensation, and demonstrate almost $3 \mathrm{~dB}$ performance improvement brought by PS-QPSK in both cases.

\section{Generation AND DeteCtion OF PS-QPSK}

\section{A. PS-QPSK: A Set-Partitioned PDM-QPSK Signal}

The PS-QPSK format is an 8-level, 3 bit per symbol linear modulation format. At each symbol time one information bit selects one of two orthogonal polarizations of the light over which a QPSK symbol, selected by the remaining 2 information bits, is encoded. Nothing is transmitted on the remaining polarization at that symbol time. Fig. 1(a) shows a PS-QPSK transmitted symbol on the complex-envelope $\mathrm{X}^{\prime}$ and $\mathrm{Y}^{\prime}$ planes: one of 4 available QPSK symbols is selected on $\mathrm{X}^{\prime}$, while zero power is transmitted on $\mathrm{Y}^{\prime}$. In the example, the black dots represent one four-dimensional (4D) symbol. An alternative representation of the PS-QPSK symbols shown in Fig. 1(b) can be obtained from 


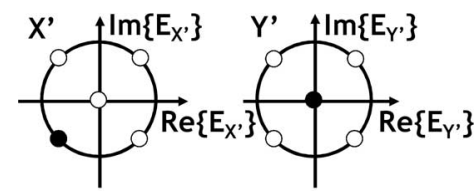

a)

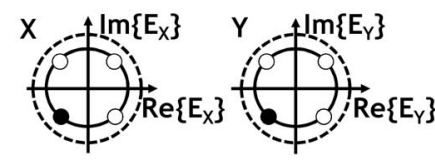

b)

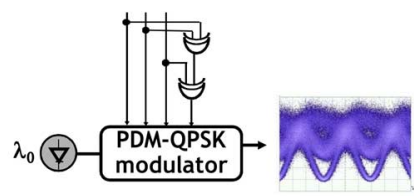

c)

Fig. 1. (a) First representation of PS-QPSK symbols, (b) second representation of PS-QPSK symbols as a subset of PDM-QPSK symbols, and (c) PS-QPSK mapping using PDM-QPSK hardware configuration.

the first representation after a change of polarization basis, i.e., after a polarization transformation. The polarization transformation bringing from the first representation in Fig. 1(a) to the representation in Fig. 1(b) is given by

$$
\left[\begin{array}{l}
E_{X} \\
E_{Y}
\end{array}\right]=\frac{1}{\sqrt{2}} \cdot\left[\begin{array}{cc}
1 & -1 \\
1 & 1
\end{array}\right] \cdot\left[\begin{array}{l}
E_{X^{\prime}} \\
E_{Y^{\prime}}
\end{array}\right]
$$

so that the symbol represented by black dots in Fig. 1(b) is the transform of the symbol in Fig. 1(a). This second representation uses the two conventional constellation diagrams of PDM-QPSK (one constant-envelope QPSK for each polarization tributary X and Y) and shows that PS-QPSK symbols may be considered as a subset of standard PDM-QPSK symbols.

With the specific signaling of PS-QPSK, not all four QPSK symbols can be used along $\mathrm{X}$ independently from the other four symbols along $\mathrm{Y}$. More specifically, the symbol on $\mathrm{X}$ is either in phase or in opposite phase with the symbol on polarization $\mathrm{Y}$, but never in quadrature (i.e., with a \pm 90 degrees phase shift), as depicted for instance in Fig. 1(b). Hence, a PS-QPSK symbol can take only 8 states out of the 16 possible states for a PDM-QPSK symbol. The particular interest of this representation stands in the fact that it provides simple guidelines for the generation of PS-QPSK out of the same hardware configuration (drivers and I/Q modulators) as PDM-QPSK. To associate the three information bits of PS-QPSK to the eight PDM-QPSK symbols subset, a simple mapping rule was proposed in [2]. As described in Fig. 1(c), this mapping rule resorts on a few XOR gates in order to correlate the four input data streams of a PDM-QPSK transmitter. In this case, PS-QPSK inherits from most of the PDM-QPSK transmitter, with the addition of a logic operation between data generated along the two orthogonal polarizations axes, creating some form of correlation. This PS-QPSK representation can be seen as an Ungerboeck-like set partitioning of the PDM-QPSK [9]. This mapping leads to an increase of the minimum Euclidean distance between the constellation points by a factor of $\sqrt{2}$ [3]. Fig. 2 shows a simpli-

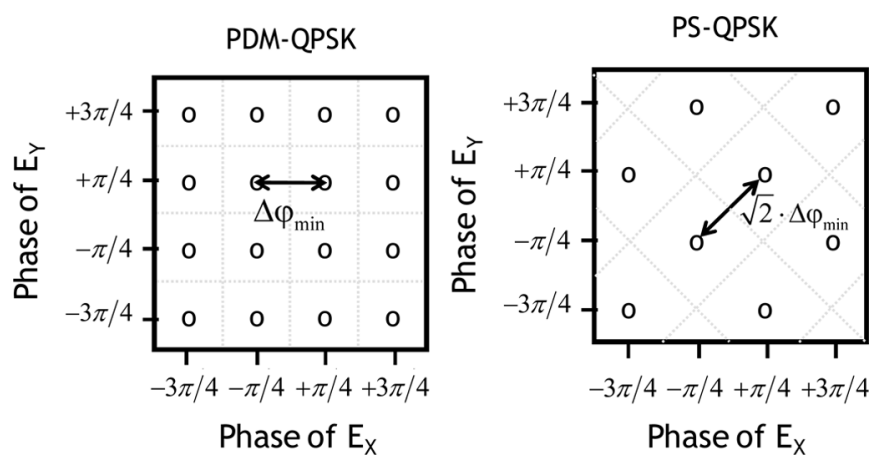

Fig. 2. Information symbols and decision regions on joint phase plane for (left) PDM-QPSK format and (right) PS-QPSK format.

fied representation of the decision regions for the 16 allowed PDM-QPSK symbols and for the 8 PDM-QPSK symbol subset (representing the 8 PS-QPSK symbols) on a joint phase plane of $\mathrm{E}_{\mathrm{X}}$ and $\mathrm{E}_{\mathrm{Y}}$, restricted modulo $2 \pi$ in each dimension. The interest of the joint phase representation is that the amplitude information is not reported, thus allowing a reduction of the 4-dimensional signal space to a 2-dimensional space with a simple visualization [5]. These joint phase plane representations clearly illustrate that PS-QPSK symbols are a set partitioning of the 16 PDM-QPSK symbols. A $\sqrt{2}$ increase in minimum phase-distance $\Delta \varphi_{\min }$ among symbols is observed, which by coincidence nicely pairs with the $\sqrt{2}$ increase in minimum Euclidean distance.

\section{B. Generation and Detection of PS-QPSK}

As the generation of PS-QPSK may resort on the same hardware configuration as PDM-QPSK, it would be tempting to reuse most of the digital signal processing used for PDM-QPSK in coherent receivers. Digital signal processing for coherent PDM-QPSK receivers commonly comprises the following steps [10], [11]: resampling, compensation of chromatic dispersion, polarization demultiplexing, frequency offset recovery, carrier phase recovery and symbol identification. However, two major shortcomings occur for the digital processing of PS-QPSK:

- The chosen correlation between the information bits carried on the two polarizations of the light implies that the state of polarization (SOP) of PS-QPSK is binary as for PDM- binary phase shift keying (BPSK), which causes the failure of the constant modulus algorithm (CMA) [12], [13], the most widely used adaptive blind equalizer for polarization-demultiplexing in PDM-QPSK systems.

- The inherent phase ambiguity derived from the use of a blind phase recovery, e.g., the 4th-power method [14], may induce phase mismatch between the two polarizations and cause catastrophic error propagation unrecoverable with differential decoding.

In the following, we propose a novel solution for each one of these two shortcomings for PS-QPSK.

1) Polarization Demultiplexing: Polarization demultiplexing is commonly realized by adaptive equalizers usually implemented in a butterfly structure with four banks of finite impulse response (FIR) filters [15]. Among all the methods to adapt the tap values of these FIR filters, the constant modulus algorithm 


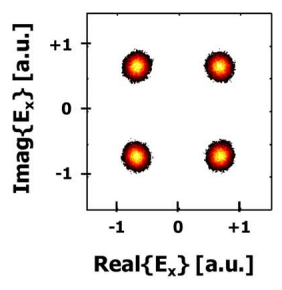

a)

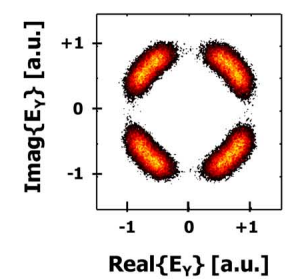

b)

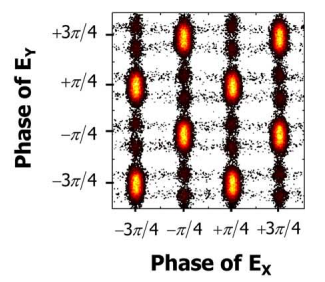

c)
Fig. 3. Example of convergence failure of CMA to demultiplex the polarization tributaries (a) $\mathrm{E}_{\mathrm{x}}$ and (b) $\mathrm{E}_{\mathrm{y}}$ of PS-QPSK, and (c) corresponding representation on the joint phase plane.

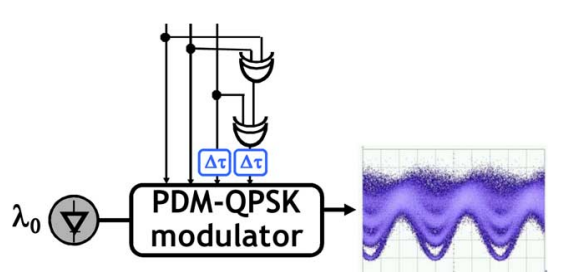

Fig. 4. Proposed alternative solution to keep using conventional CMA to demultiplex the polarization tributaries $\mathrm{E}_{\mathrm{x}}$ and $\mathrm{E}_{\mathrm{y}}$ of PS-QPSK signals, and corresponding eye diagram of the generated signal.

(CMA) and the least mean square phase locked loop (LM-PLL) algorithm are considered as the most favourable techniques. For the realization of coherent PDM-QPSK receivers using application specific integrated circuits, the massive parallelization of operations makes the CMA more appealing than the LMS-PLL algorithm which requires long feedback paths from the frequency and phase carrier recovery stage. However, the CMA cannot be used in PS-QPSK systems. Indeed, the two polarization tributaries of PS-QPSK signals being correlated, the constant modulus criterion becomes insufficient to obtain a proper polarization demultiplexing [16]. As depicted in the example of 3-a) and 3-b), the two polarizations of the PS-QPSK signal cannot be correctly recovered together by the CMA, and the resulting joint phase plane representation in Fig. 3(c) illustrates the failure of the detected phase of the signal after carrier phase recovery.

Some modified versions of the CMA have been recently proposed to allow polarization demultiplexing and equalization of PS-QPSK [16], [17]. We propose here an alternative solution to solve the issue of polarization demultiplexing while keeping the conventional CMA. As shown in Fig. 4, our idea consists in shifting in time by a few symbols delay $\Delta \tau$ the correlated data streams driving the $\mathrm{I} / \mathrm{Q}$ modulators on $\mathrm{X}$ and $\mathrm{Y}$ polarizations of the PS-QPSK transmitter. Such a delay introduces a fake independency between the polarization tributaries and makes the PS-QPSK signal statistically equivalent to a PDM-QPSK signal as depicted in Fig. 4. Therefore, a T/2-spaced adaptive equalizer with $\mathrm{N}$-taps based on the conventional CMA can properly demultiplex this signal pending that the introduced delay is greater than N/2. After polarization demultiplexing, the true PS-QPSK signal is recovered by removing digitally the introduced symbol delay between the polarizations. The actual value used for time alignment between the polarizations may actually depend on the response of the CMA, which can introduce some extra delay under certain operating conditions.
This possible extra delay is not an issue for the proposed solution as it is inherent of most of coherent systems employing polarization division multiplexing and is usually fixed during the initialization process in practical systems.

2) Phase Locking: After polarization demultiplexing, the phase offsets of the QPSK signals on the two polarization tributaries are independently estimated using feed-forward carrier phase recovery schemes. For instance, the estimated phase offset of polarization X derived from the 4th-power Viterbi and Viterbi technique [14] is expressed as:

$$
\theta_{x}=\frac{1}{4} \arg \left[\sum_{k=-N}^{+N}\left|E_{x}(k)\right|^{2} \cdot\left(e^{j \phi_{x}(k)}\right)^{4}\right]
$$

where $\phi_{\mathrm{x}}(\mathrm{k})$ is the phase of the received field at the kth symbol $\mathrm{E}_{\mathrm{x}}(\mathrm{k})$ affected by the information phase, the phase offset and the phase noise (both induced by additive white Gaussian noise (AWGN) and possibly coming from the line). The field phase is raised to the power of 4 to remove the QPSK information phase. The amplitude is preferably raised to the power of 2 instead of 4 in order not to excessively enhance additive noise, as prescribed by Viterbi. The average over $2 \mathrm{~N}+1$ samples smoothes AWGN induced phase fluctuations on the field. At the output of the carrier recovery block, the estimated phase offset is of the form:

$$
\theta_{x}(k)=\psi_{x}(k)+n_{x} \cdot \frac{\pi}{2}
$$

where $-\pi / 4<\psi_{\mathrm{x}}<\pi / 4$ is the estimated offset modulo $\pi / 2$, and $n_{\mathrm{x}}$ is an integer accounting for the intrinsic ambiguity of the blind estimation. A similar estimation is performed for the Y offset leading:

$$
\theta_{y}(k)=\psi_{y}(k)+n_{y} \cdot \frac{\pi}{2}
$$

with $n_{y}$ independent of $n_{x}$.

We will call (nx, ny) the phase-ambiguity factors. After subtraction of the estimated phase offset, the recovered phase of $\mathrm{X}$ is $\varphi_{\mathrm{x}}=\phi_{\mathrm{x}}-\theta_{\mathrm{x}}$, and that of $\mathrm{Y}$ is $\varphi_{\mathrm{y}}=\phi_{\mathrm{y}}-\theta_{\mathrm{y}}$.

Generally, the phase-ambiguity inherent from the use of the Mth-power elevation technique is compensated by the use of differential coding/decoding. For PDM-QPSK signals, having different phase-ambiguity factors $\left(\mathrm{n}_{\mathrm{x}}=2, \mathrm{n}_{\mathrm{y}}=1\right.$ for instance) on $\mathrm{X}$ and $\mathrm{Y}$ is not an issue. Maximum likelihood decisions are usually drawn independently on each polarization since information carried on $\mathrm{X}$ and $\mathrm{Y}$ polarizations are independent. However, in the case of PS-QPSK, data symbols carried on X and Y polarizations are correlated. In the absence of noise, the phase information should satisfy the following relation:

$$
\varphi_{x}(k)-\varphi_{y}(k)=m \cdot \pi
$$

with $\mathrm{m}$ being an integer.

Ensuring this relationship is equivalent to forcing the differential phase ambiguity $\varepsilon$ to be a multiple of $\pi$ :

$$
\varepsilon=\left(n_{x}-n_{y}\right) \cdot \frac{\pi}{2}=m \cdot \pi
$$

This is realized using a phase ambiguity detector as depicted in Fig. 5. This detector combines the polarization tributaries by 


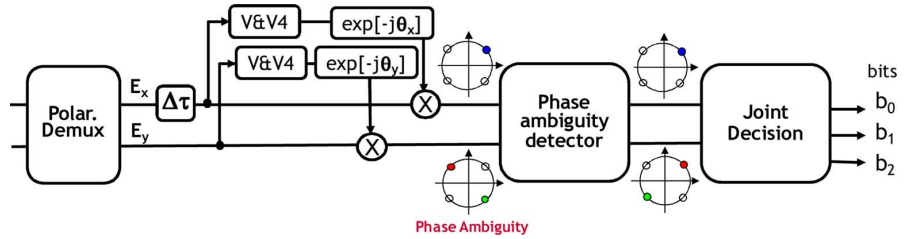

Fig. 5. Scheme of new coherent receiver for PS-QPSK signals. "V\&V4" stands for the Viterbi and Viterbi algorithm with elevation to the 4th power.

forming the field $\mathrm{Ex} \cdot \mathrm{Ey}^{*}$ (where $*$ stands for conjugate), and its phase $\phi_{\mathrm{x}}-\phi_{\mathrm{y}}$, which in noiseless PS-QPSK ideally takes only 0 and $\pi$ values, is estimated with a 2nd power Viterbi and Viterbi algorithm [14]:

$$
\theta_{\Delta}=\frac{1}{2} \arg \left[\sum_{k=-L}^{L}\left|E_{x}(k) \cdot E_{y}^{*}(k)\right|^{2}\right]
$$

This value is smoothed out and rounded to the nearest $\pi / 2$ value in order to force the detected differential phase ambiguity $\varepsilon$ to be a multiple of $\pi$. The detected differential phase ambiguity is then subtracted on the field of one polarization and the PS-QPSK enters in the decision block, in which differential decoding can be applied as for standard PDM-QPSK.

Denote now the generic 4-dimensional PS-QPSK symbol as a real vector $\mathrm{E}=\left[\mathrm{E}_{\mathrm{xr}}, \mathrm{E}_{\mathrm{xi}}, \mathrm{E}_{\mathrm{yr}}, \mathrm{E}_{\mathrm{yi}}\right] \varepsilon \mathrm{R}^{4}$, where we decomposed $E_{\mathrm{x}}=\mathrm{E}_{\mathrm{xr}}+\mathrm{j} . \mathrm{E}_{\mathrm{xi}}$ in its real and imaginary components, and similarly for $\mathrm{E}_{\mathrm{y}}$. The squared Euclidean distances $\mathrm{d}(\mathrm{k})^{2}$ between the received field vector $E_{r}$ and the symbol field vector $E_{k}$ corresponding to the kth PS-QPSK constellation point is given by

$$
d^{2}(k)=\sum_{i=1}^{4}\left[\vec{E}_{r}(i)-\hat{E}_{k}(i)\right]^{2}
$$

Thus, the Euclidean distance of the field vector $E_{\mathrm{r}}$ at the input of the decision block is calculated with respect to all 8 PS-QPSK symbol field vectors, and the closest symbol, along with its bitmapping, is decided.

Fig. 6 summarizes the DSP used for coherent detection of the PS-QPSK signals in the following sections. Fig. 6(a) depicts the novel DSP used when decorrelating the polarization tributaries of the PS-QPSK signal. We refer to this signal as scrambled PS-QPSK. The DSP involves the conventional CMA algorithm for polarization demultiplexing followed by polarization time alignment, phase tracking followed by phase locking (including the phase ambiguity detector) and the joint Euclidean distance decision before error counting based on differential decoding. Fig. 6(b) depicts the DSP used when the polarization tributaries of the PS-QPSK signal are time-aligned. We refer to this signal as time-aligned PS-QPSK. This DSP differs from the previous one as a LMS-PLL algorithm involving a phase locked loop replaces the CMA for polarization demultiplexing. Although the LMS-PLL algorithm is less appealing than CMA for the realization of coherent receivers, the LMS criterion for convergence allows proper demultiplexing of PS-QPSK [18] and will be used as a reference for comparison.

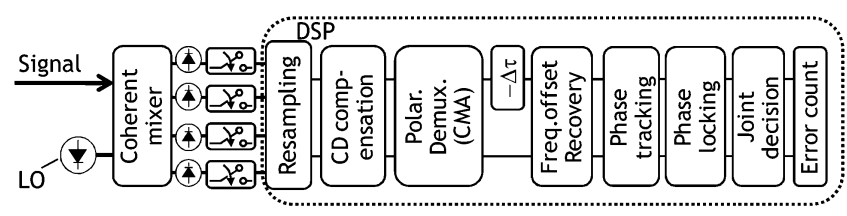

a)

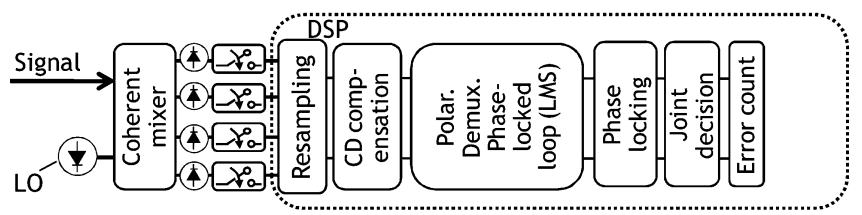

b)

Fig. 6. Scheme of the proposed coherent receiver for scrambled PS-QPSK signals, and (b) scheme of the coherent receiver for time-aligned PS-QPSK signals.

\section{BACK-TO-BACK CHARACTERIZATION}

To generate PS-QPSK data, the light from the test laser source is divided into two branches, sent to two distinct QPSK modulators (having $3 \mathrm{~dB}$-bandwidth in the range of $25-30 \mathrm{GHz}$ ) operating at 28 Gbaud, before polarization combining to form PS-QPSK data. The four input data streams driving the two I-Q modulators are correlated $2^{15}-1$-bit-long binary sequences provided by a field programmable gate array (FPGA) board according to Fig. 1(a). A tuneable optical delay line has been introduced on one branch to generate either time-aligned PS-QPSK or scrambled PS-QPSK by temporally decorrelating the two polarization tributaries by a few symbols. We chose to introduce a delay of 7 symbols, which is larger than the correlation time of the 13-tap T/2-spaced adaptive equalizer used in the following experiments.

Fig. 7(a) depicts the setup used for the experimental measurement of sensitivity to optical noise and Fig. 7(b) shows an example of constellation of the received PS-QPSK signal with an OSNR of $11 \mathrm{~dB} / 0.1 \mathrm{~nm}$. The corresponding phase plane representation is depicted in Fig. 7(c) together with the phase plane of the received PDM-QPSK at the same OSNR for comparison. On top of demonstrating the ability of the proposed technique to re-use the standard CMA, this figure clearly illustrates the higher tolerance of PS-QPSK compared to PDM-QPSK against optical noise.

Fig. 8 shows the measured $\mathrm{Q}^{2}$-factor performance versus OSNR of time-aligned and scrambled PS-QPSK compared to PDM-QPSK at 28 Gbaud. Triangles and squares represent the results obtained when the test channel is scrambled and time-aligned PS-QPSK respectively. Circles represent the results obtained when the test channel is PDM-QPSK modulated. These results demonstrate that the scrambled PS-QPSK performs similarly to the time-aligned PS-QPSK. No convergence failure was observed with the CMA for the scrambled PS-QSPK, thus validating the interest of the proposed technique. These curves also demonstrate a better sensitivity to noise of $\sim 2.7 \mathrm{~dB}$ brought by the four-dimensional PS-QPSK format with respect to PDM-QPSK at a $\mathrm{Q}^{2}$-factor of $10 \mathrm{~dB}$. Out of this 2.7-dB improvement, $1.25 \mathrm{~dB}$ stands for the bit rate difference $\left(10 \cdot \log _{10}(112 / 84)\right)$. The $1.45 \mathrm{~dB}$ improvement in OSNR sensitivity brought by PS-QPSK with respect to 


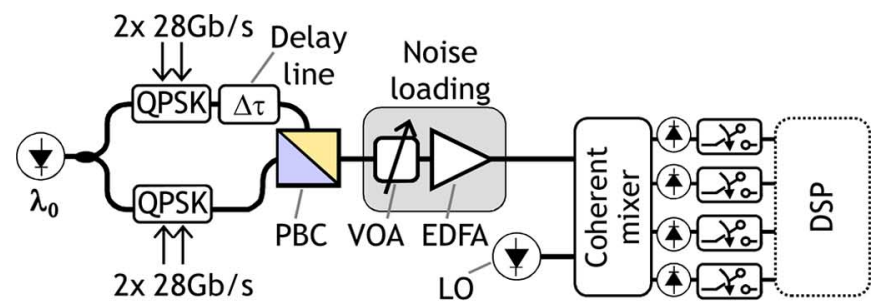

a)
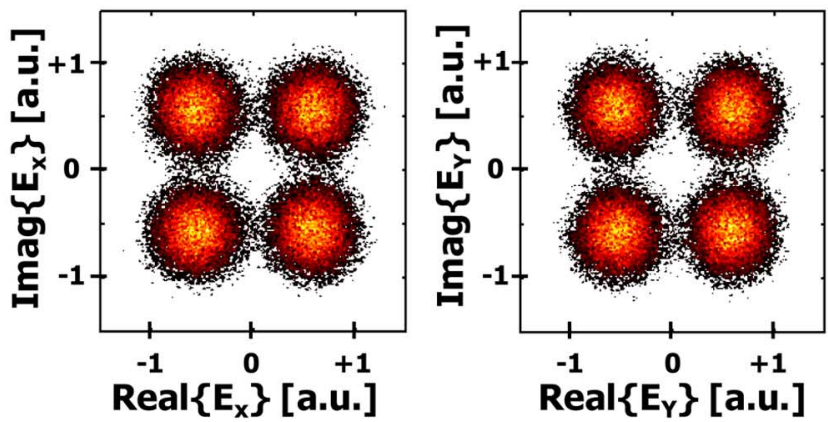

b)

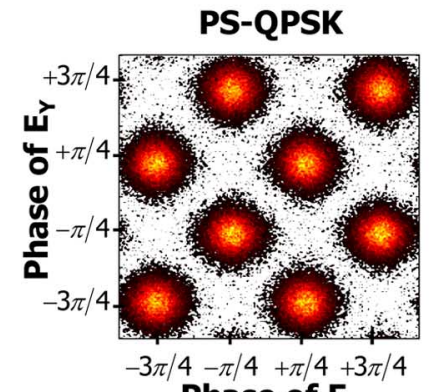

Phase of $E_{x}$

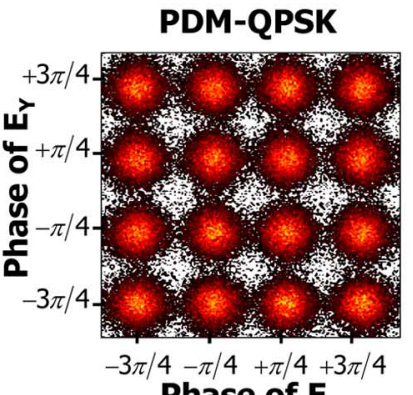

Phase of $\mathbf{E}_{\mathbf{x}}$ c)

Fig. 7. (a) Experimental setup for back-to-back characterization, (b) example of constellation of the received PS-QPSK signal with an OSNR of $11 \mathrm{~dB} / 0.1$ $\mathrm{nm}$ and (c) corresponding phase plane representations of PS-QPSK and PDMQPSK for this OSNR.

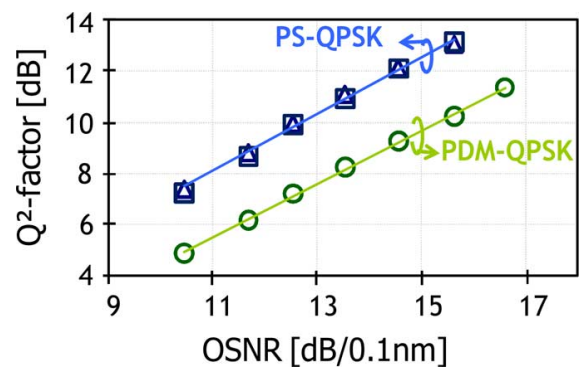

Fig. 8. Measurement results on sensitivity to optical noise of scrambled (triangles) and time-aligned (squares) PS-QPSK at 28 Gbaud compared with that of PDM-QPSK (circles) at the same baud rate.

PDM-QPSK is slightly higher than that reported in [6], which we attributed to the use of differential decoding instead of direct decoding and to an enhanced tolerance to imperfect response and limited bandwidth of transmitter and receiver used in this experiment.

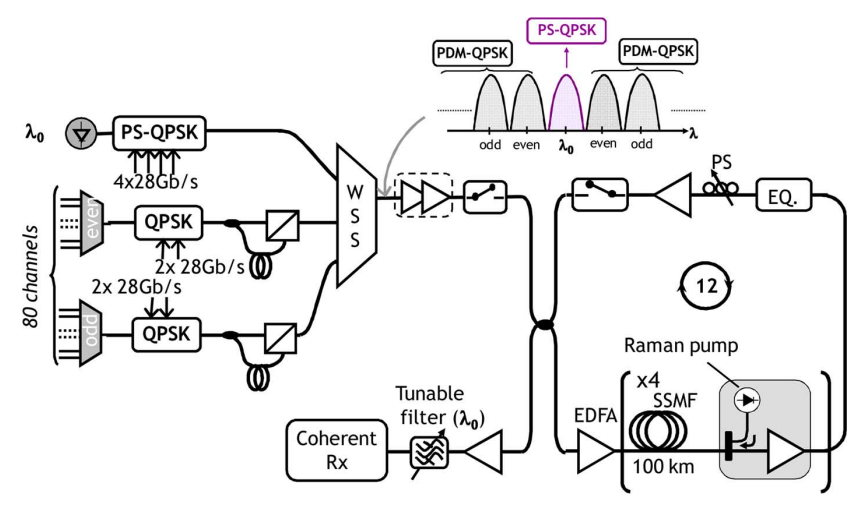

Fig. 9. Experimental setup for WDM transmission of 28-Gbaud PS-QSPK over a non dispersion managed link.

\section{TRANSMISSION EXPERIMENTS USING PS-QPSK}

\section{A. Non Dispersion-Managed Transmission}

Next, we investigated the performance of PS-QPSK in a non dispersion managed WDM transmission. As depicted in Fig. 9, our transmitter consists of 80 distributed feedback (DFB) lasers, spaced by $50 \mathrm{GHz}$ and separated into two independently modulated, spectrally interleaved even and odd combs, plus one tuneable laser (test channel), at $1546.12 \mathrm{~nm}$. The light from even and odd combs is sent to two distinct QPSK modulators operating at $28 \mathrm{Gbaud}$ (or $56 \mathrm{~Gb} / \mathrm{s}$ ). The modulators are fed by $2^{15}-1$-bitlong sequences at $28 \mathrm{~Gb} / \mathrm{s}$. Polarization multiplexing is finally performed by dividing the QPSK data into two tributaries and recombining them into a Polarisation Beam Combiner (PBC) with an approximate 300-symbol delay, yielding PDM-QPSK data at $112 \mathrm{~Gb} / \mathrm{s}$. The $28 \mathrm{Gbaud}$ PS-QPSK test channel, together with even and odd PDM-QPSK channel combs are respectively sent to input ports 1,2 and 3 of a $50 \mathrm{GHz}$ grid wavelength selective switch (WSS). Depending on the configuration of the WSS, the output multiplex can be either a PDM-QPSK multiplex with the PS-QPSK test channel inserted in the middle of the C-band as shown in the inset of Fig. 9, or a full PDM-QPSK multiplex.

As shown in Fig. 9, the resulting multiplex is boosted through a dual-stage erbium doped fiber amplifier (EDFA) and sent into the re-circulating loop which incorporates four $100 \mathrm{~km}$-long spans of SSMF. No dispersion-compensating fibre is used here. All chromatic dispersion is compensated digitally in the coherent receiver. Fibre loss is compensated for by hybrid RamanErbium optical repeaters. The Raman pre-amplifier is designed to provide $\sim 10 \mathrm{~dB}$ on-off gain. Power equalization is performed thanks to a dynamic gain equalizer (DGE) inserted at the end of the loop, just before a loop-synchronous polarization scrambler (PS). In all experiments, we measure the performance after twelve loop round-trips, i.e., after a 4800 km-long transmission. We vary the power per channel, Pch, at each fiber input by changing the output power of the amplifiers from $12 \mathrm{dBm}$ to $19 \mathrm{dBm}$ while keeping the number of channels constant. Since the maximum output power of the amplifiers is $19 \mathrm{dBm}$ we reduce the number of channels to extend the studied power range, when necessary. In all experiments, we chose to set the launch 


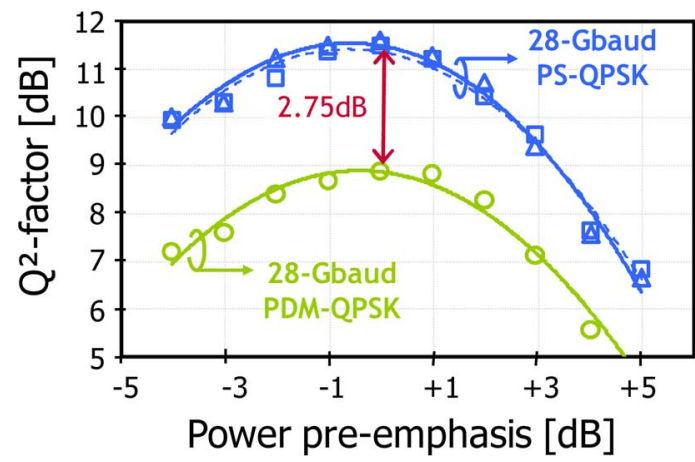

Fig. 10. Experimental results of scrambled (triangles) and time-aligned (squares) PS-QPSK at 28 Gbaud compared with that of PDM-QPSK (circles) at the same baud rate over a non dispersion managed WDM transmission link of $4800 \mathrm{~km}$

power of the test channel at the same level as all the co-propagating channels. At the receiver side, the channel under study $(1546.12 \mathrm{~nm})$ is selected by a $0.4 \mathrm{~nm}$ bandwidth filter and sent to the coherent receiver.

Fig. 10 shows the $\mathrm{Q}^{2}$-factor of the test channel versus its power, normalized to the power yielding the optimum $\mathrm{Q}^{2}$-factor for PDM-QPSK modulation, i.e., $-3 \mathrm{dBm}$. Triangles and squares represent the results obtained when the test channel is scrambled and time-aligned PS-QPSK respectively. Circles represent the results obtained when the test channel is PDM-QPSK modulated. Similar performance is observed between the scrambled and time-aligned PS-QPSK signals, indicating that the proposed method to keep using CMA introduces no penalty after WDM propagation. For power pre-emphasis below $0 \mathrm{~dB}$, we can observe a $\mathrm{Q}^{2}$-factor improvement of about $3 \mathrm{~dB}$ when moving from PDM-QPSK to PS-QPSK modulated signals, in line with back-to-back characterization as well as numerical results obtained in [5]. For power pre-emphasis greater than $+3 \mathrm{~dB}$, we observe that the benefit brought by the PS-QPSK format is significantly decreasing. However the power corresponding to the optimum performance is nearly the same in both cases and PS-QPSK still outperforms PDM-QPSK by about $2.75 \mathrm{~dB}$. The PS-QPSK format achieves slightly more than $11.5 \mathrm{~dB} \mathrm{Q}^{2}$-factor performance while the PDM-QPSK format approaches the FEC limit of $8.5 \mathrm{~dB}$ (i.e., a $4 \cdot 10^{-3}$ bit error ratio). Besides, over the same transmission link, we reported experimentally in [18] that PDM-QPSK achieves approximately the same $\mathrm{Q}^{2}$-factor performance $(\sim 11.2 \mathrm{~dB})$ after a $2400 \mathrm{~km}$-long transmission. This indicates that PS-QPSK can achieve twice as much distance as PDM-QPSK while reaching the same performance.

\section{B. Dispersion-Managed Transmission}

We finally study the performance of PS-QPSK impact over optically dispersion managed systems. As depicted in Fig. 11, fibre loss is now compensated for by double stage EDFA repeaters. Each repeater includes a dispersion compensation fiber in order to partially compensate for the accumulated chromatic dispersion. To vary the dispersion map, a variable dispersion compensating module was introduced at the end of the recirculating loop to vary the residual dispersion per span. Two disper-

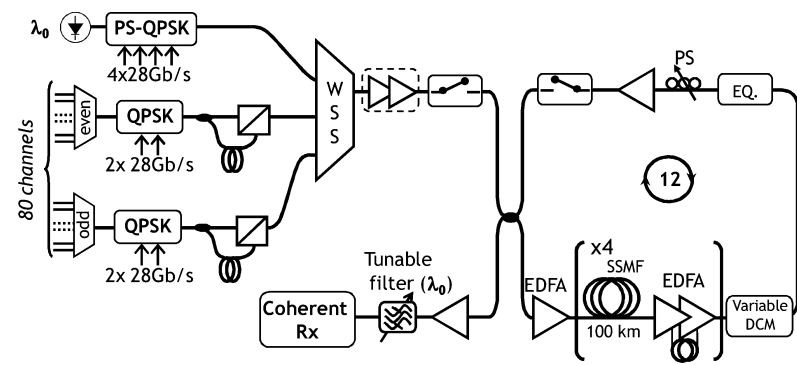

Fig. 11. Experimental setup for WDM transmission of 28-Gbaud PS-QSPK over a dispersion managed link with variable dispersion map.

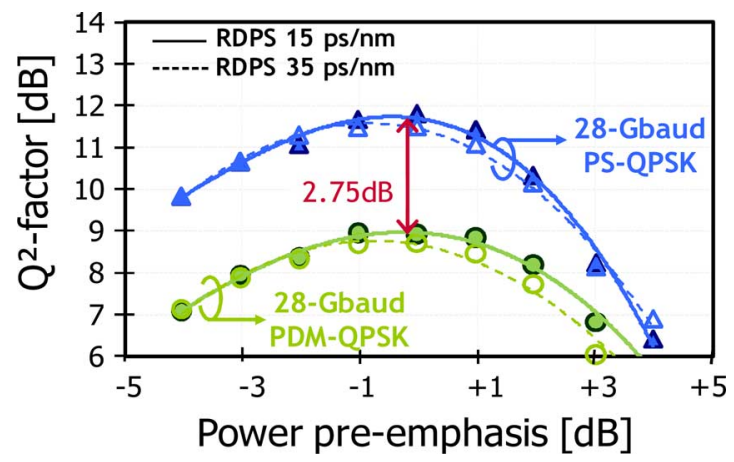

Fig. 12. Experimental results of scrambled PS-QPSK (triangles) at 28 Gbaud compared with that of PDM-QPSK (circles) at the same baud rate over a dispersion managed WDM transmission link of $2000 \mathrm{~km}$ with variable dispersion map.

sion maps having respectively a residual dispersion per span of $15 \mathrm{ps} / \mathrm{nm}$ and $35 \mathrm{ps} / \mathrm{nm}$ were investigated.

Fig. 12 shows the $\mathrm{Q}^{2}$-factor of the test channel after a 2000 $\mathrm{km}$-long WDM transmission versus its power, normalized to the power yielding the optimum $\mathrm{Q}^{2}$-factor for PDM-QPSK modulation. Empty and solid triangles (circles) represent the results obtained when the test channel is scrambled PS-QPSK (resp. PDM-QPSK) with a residual dispersion per span of 15 and $35 \mathrm{ps} / \mathrm{nm}$ respectively. As in the case of non-dispersion managed transmission link, the power corresponding to the optimum performance is nearly the same for PS-QPSK and PDM-QPSK whatever the residual dispersion per span. The impact of the variable residual dispersion per span appears smaller for PS-QPSK than for PDM-QPSK and PS-QPSK outperforms PDM-QPSK by almost $3 \mathrm{~dB}$ in both cases, confirming the potential of PS-QPSK over dispersion managed systems.

All these results demonstrate that, at the same baud rate operation, PS-QPSK provides substantial performance gains over PDM-QPSK which could be of high interest for on-the-fly fall-back solution to PDM-QPSK depending on channel impairments both for dispersion managed and non-dispersion managed systems.

\section{CONCLUSION}

In this article, we have experimentally demonstrated longhaul transmission of 28 Gbaud PS-QPSK signal in WDM systems with electrical and optical dispersion compensation. We have proposed and demonstrated a novel implementation for optical PS-QPSK transceivers enabling to keep most of standard coherent PDM-QPSK transceivers. We have also compared 
the performance of PS-QPSK with that of PDM-QPSK at 28 Gbaud and demonstrated that PS-QPSK achieves $3 \mathrm{~dB}$ higher $\mathrm{Q}^{2}$-factor performance over dispersion managed and non-managed transmission links. Pending a 25\% reduction of the transmitted capacity, PS-QPSK could allow to keep operating on transmission links where conventional $100 \mathrm{~Gb} / \mathrm{s}$ PDM-QPSK fails. This demonstrates the potential of this solution to be considered an attractive complement to the formats already proposed for software-defined optical transceivers.

\section{REFERENCES}

[1] H. Buelow, "Polarization QAM modulation for coherent detection schemes," in Proc. OFC, San Diego, CA, 2009, paper OWG2.

[2] M. Karlsson and E. Agrell, "Which Is the most power-efficient modulation format in optical links?," Opt. Exp., vol. 17, pp. 10814-10819, 2009.

[3] E. Agrell and M. Karlsson, "Power-efficient modulation formats in coherent transmission systems," J. Light.w Technol., vol. 27, no. 22, pp. 5115-5126, Nov. 2009.

[4] M. Sjödin et al., "Comparison of polarization-switched QPSK and polarization-multiplexed QPSK at $30 \mathrm{Gbit} / \mathrm{s}$," Opt. Exp., vol. 19, no. 8, p. 7839, 2011.

[5] P. Serena, A. Vannucci, and A. Bononi, "The performance of polarization switched-QPSK (PS-QPSK) in dispersion managed WDM transmissions," in Proc. ECOC, Brussels, Belgium, 2010, paper Th.10.E.2.

[6] P. Poggiolini, G. Bosco, A. Carena, V. Curri, and F. Forghieri, "Performance evaluation of coherent WDM PS-QPSK (HEXA) accounting for non-linear fiber propagation effects," Opt. Exp., vol. 18, pp. 11360-11371, 2010.

[7] O. Rival, G. Villares, and A. Morea, "Impact of inter-channel nonlinearities on the planning of $25-100 \mathrm{~Gb} / \mathrm{s}$ elastic optical networks," $J$. Lightw. Technol., vol. 29, no. 9, pp. 1326-1334, May. 2011.
[8] J. Renaudier, O. Bertran-Pardo, H. Mardoyan, M. Salsi, P. Tran, E. Dutisseuil, G. Charlet, and S. Bigo, "Experimental comparison of 28 gbaud polarization switched- and polarization division multiplexedQPSK in WDM long-haul transmission system," in Proc. ECOC, Geneva, Switzerland, 2011, paper Mo.2.B.3.

[9] G. Ungerboeck, "Channel coding with multilevel/phase signals," IEEE Trans. Inf. Theory, vol. IT-28, no. 1, pp. 55-67, Jan. 1982.

[10] C. R. S. Fludger, T. Duthel, D. van den Borne, C. Schulien, E.-D. Schmidt, T. Wuth, J. Geyer, E. De Man, G.-D. Khoe, and H. de Waardt, "Coherent equalization and POLMUX-RZ-DQPSK for robust 100-GE transmission," J. Lightw. Technol., vol. 26, no. 1, pp. 64-72, Jan. 2008.

[11] J. Renaudier, O. Bertran-Pardo, G. Charlet, M. Salsi, H. Mardoyan, P. Tran, and S. Bigo, " $8 \mathrm{~Tb} / \mathrm{s}$ long haul transmission over low dispersion fibers using $100 \mathrm{~Gb} / \mathrm{s}$ PDM-QPSK channels paired with coherent detection," Bell Labs Tech. J., vol. 14, no. 4, pp. 27-45, 2010.

[12] M. Yan, Z. Tao, H. Zhang, W. Yan, T. Hoshida, and J. C. Rasmussen, "Adaptive blind equalization for optical BPSK systems," in Proc. ECOC, Italy, 2010, paper Th.9.A.4.

[13] S. Haykin, Unsupervised Adaptive Filtering. New York: Wiley, 2000.

[14] A. J. Viterbi and A. M. Viterbi, "Nonlinear estimation of PSK-modulated carrier phase with application to burst digital transmission," IEEE Trans. Inf. Theory, vol. IT-29, no. 4, pp. 543-551, Jul. 1983.

[15] S. J. Savory, "Digital filters for coherent optical receivers," Opt. Exp., vol. 16, no. 2, pp. 804-817, Jan. 2008.

[16] P. Johannisson, M. Sjodin, M. Karlsson, H. Wymeersch, E. Agrell, and P. A. Andrekson, "Modified constant modulus algorithm for polarization-switched QPSK," Opt. Exp., vol. 19, no. 8, p. 7734, 2011.

[17] D. S. Millar and S. J. Savory, "Blind adaptive equalization of polarization-switched QPSK modulation," Opt. Exp., vol. 19, no. 9, pp. 8533-8538, 2011.

[18] L. E. Nelson, X. Zhou, N. Mac Suibhne, A. D. Ellis, and P. Magill, "Experimental comparison of coherent polarization-switched QPSK to polarization-multiplexed QPSK for $10 \times 100 \mathrm{~km}$ WDM transmission," Opt. Exp., vol. 19, no. 11, p. 10849, 2011.

[19] J. Renaudier, O. Bertran-Pardo, P. Tran, L. Pierre, H. Mardoyan, G. Charlet, and S. Bigo, "Nonlinear tolerance of ultra-densely spaced 100 Gb/s coherent PDM-QPSK channels," in Proc. ECOC, Torino, Italy, 2010, paper Mo.2.C.3. 